FACTA UNIVERSITATIS (NIŠ)

Ser. Math. Inform. Vol. 35, No 4 (2020), 1049-1057

https://doi.org/10.22190/FUMI2004049S

\title{
SOME CHARACTERIZATIONS OF THREE-DIMENSIONAL $f$-KENMOTSU RICCI SOLITONS
}

\author{
Avijit Sarkar and Pradip Bhakta
}

(C) 2020 by University of Niš, Serbia | Creative Commons Licence: CC BY-NC-ND

Abstract. The aim of the present paper is to give some characterizations of $f$-Kenmotsu Ricci soliton with a supporting example.

Keywords: $f$-Kenmotsu manifold; Ricci almost soliton; gradient Ricci soliton.

\section{Introduction}

The revolutionary concept of Ricci flow was introduced by Hamilton [5] in order to solve Poincare conjecture. The conjecture was fully solved by Perelman [11] using Hamilton's Ricci flow technique. After the work of Perelman, the study of Ricci flow has become an important topic in differential geometry. A Ricci flow is a weak parabolic heat type partial differential equation of the following form

$$
\begin{gathered}
\frac{\partial g_{i j}}{\partial t}=-2 S_{i j}, \\
g(0)=g_{0} .
\end{gathered}
$$

Here $g_{i j}$ denotes the components of Riemannian metric $g$ and $S_{i j}$ denotes the components of Ricci tensor $S$. A Ricci soliton is a solution of the above equation which is constant up to diffeomorphism and scaling. A Ricci soliton on a Riemannian manifold is characterized by the equation

$$
\left(£_{V} g\right)(X, Y)+2 S(X, Y)+2 \lambda g(X, Y)=0 .
$$

Here $\lambda$ is a constant, called soliton constant and the vector field $V$ is called soliton vector field. A Ricci soliton is called expanding, shrinking or steady while $\lambda$ is positive, negative or zero. A Ricci soliton is called Ricci almost soliton if $\lambda$ is

Received August 25, 2020; accepted October 07, 2020

2020 Mathematics Subject Classification. Primary 53 C25; Secondary 53 D 15. 
considered as a function instead of a constant [12]. A Ricci soliton is called gradient Ricci soliton if the soliton vector field is gradient of a potential function [13]. The study of Ricci solitons on almost contact manifolds was first initiated by Ramesh Sharma [16]. The Ricci solitons on almost contact manifolds have been studied by several authors ([4], [13], [15]). Ricci soliton on $(\kappa, \mu)$ contact metric manifold has been studied by the present authors in [14]

The notion of Kenmotsu manifold was introduced by K. Kenmotsu and was subsequently generalized to $f$-Kenmotsu manifolds. For details we refer to [8] and [9]. Ricci solitons on Kenmotsu manifold have been studied in [6]. The notion of $\phi$-Ricci symmetric manifolds was introduced by U. C. De and A. Sarkar [2]. The notion of $\phi$-symmetric manifolds was introduced by T. Takahashi [17]. Later several authors studied $\phi$-symmetric manifolds. Three dimensional quasi-Sasakian manifolds with cyclic parallel and $\eta$-parallel Ricci tensor have been studied by $\mathrm{U}$. C. De and A. Sarkar [3].

The objective of the present paper is to give some characterizations of $f$-Kenmotsu manifolds with Ricci solitons and hence establish the relations between such manifolds with locally $\phi$-symmetric manifolds and manifolds with cyclic parallel and $\eta$-parallel Ricci tensors.

The present paper is organised as follows:

After the introduction, we give will required preliminaries in Section 2. In Section 3 , we will study three dimensional $f$-Kenmotsu manifolds admitting Ricci soliton. Section 4 contains a supporting example.

\section{Preliminaries}

An odd dimensional smooth manifold $M$ is said to be an almost contact metric manifold, if there exists a $(1,1)$ tensor field $\phi$, a vector field $\xi$, a 1-form $\eta$, and a Riemannian metric $g$ on $M$ such that [1]

$$
\begin{gathered}
\phi^{2} X=-X+\eta(X) \xi, \quad \eta(\xi)=1, \quad \phi \xi=0, \quad \eta(\phi(X))=0 . \\
g(\phi X, \phi Y)=g(X, Y)-\eta(X) \eta(Y)
\end{gathered}
$$

for any vector fields $X, Y \in \chi(M)$. Such a manifold of dimension $(2 \mathrm{n}+1)$ is denoted by $M^{2 n+1}(\phi, \xi, \eta, g)$. Also $M^{2 n+1}(\phi, \xi, \eta, g)$ is called an $f$-Kenmotsu manifold if the covariant differentiation of $\phi$ satisfies

$$
\left(\nabla_{X} \phi\right) Y=f(g(\phi X, Y) \xi-\eta(Y) \phi X),
$$

where $f \in C^{\infty}(\mathrm{M})$ is such that $d f \wedge \eta=0$ ([8], [9]). If $f=\beta$ is nonzero constant, then the manifold is a $\beta$-Kenmotsu manifold [7]. If $f=0$, then the manifold is cosymplectic [7]. An $f$-Kenmotsu manifold is said to be regular if $f^{2}+f^{\prime} \neq 0$, where $f^{\prime}=\xi f$. For an $f$-Kenmotsu manifold, it follows from (2.3)

$$
\nabla_{X} \xi=f(X-\eta(X) \xi)
$$


The condition $d f \wedge \eta=0$ holds only for $\operatorname{dim} M \geq 5$ [10]. In a three dimensional $f$-Kenmotsu manifold, we have

$$
\begin{aligned}
& R(X, Y) Z=\left(\frac{r}{2}+2 f^{2}+2 f^{\prime}\right)(X \wedge Y) Z \\
&-\left(\frac{r}{2}+3 f^{2}+3 f^{\prime}\right)\{\eta(X)(\xi \wedge Y) Z+\eta(Y)(X \wedge \xi) Z\} \\
& S(X, Y)=\left(\frac{r}{2}+f^{2}+f^{\prime}\right) g(X, Y)-\left(\frac{r}{2}+3 f^{2}+3 f^{\prime}\right) \eta(X) \eta(Y), \\
& Q X=\left(\frac{r}{2}+f^{2}+f^{\prime}\right) X-\left(\frac{r}{2}+3 f^{2}+3 f^{\prime}\right) \eta(X) \xi
\end{aligned}
$$

where $(X \wedge Y) Z=g(Y, Z) X-g(X, Z) Y$, also $R, S$ and $r$ are Riemannian curvature tensor, Ricci curvature tensor and scalar curvature on $M$ respectively [9]. From (2.5) and (2.6) we get

$$
\begin{gathered}
R(X, Y) \xi=-\left(f^{2}+f^{\prime}\right)(\eta(Y) X-\eta(X) Y) \\
S(X, \xi)=-2\left(f^{2}+f^{\prime}\right) \eta(X) \\
S(\xi, \xi)=-2\left(f^{2}+f^{\prime}\right) \\
Q \xi=-2\left(f^{2}+f^{\prime}\right) \xi
\end{gathered}
$$

As a consequence of (2.4), we also have

$$
\left(\nabla_{X} \eta\right)(Y)=f g(\phi X, \phi Y) .
$$

Also from (2.9) it follows that

$$
S(\phi X, \phi Y)=S(X, Y)+2\left(f^{2}+f^{\prime}\right) \eta(X) \eta(Y)
$$

for all vector fields $X, Y \in \chi(M)$.

An $f$-Kenmotsu manifold $M^{(2 n+1)}(\phi, \xi, \eta, g)$ is said to be $\phi$-symmetric if its curvature tensor $R$ bears the condition

$$
\phi^{2}\left(\nabla_{X} R\right)(Y, Z) W=0,
$$

for all vector fields $X, Y, Z, W \in \chi(M)$ [17]. In particular, if $X, Y, Z, W$ are orthogonal to $\xi$, then $M^{(2 n+1)}(\phi, \xi, \eta, g)$ is said to be locally $\phi$-symmetric. An $f$-Kenmotsu manifold $M^{(2 n+1)}(\phi, \xi, \eta, g)$ is said to be $\phi$-Ricci symmetric if its Ricci operator $Q$ bears the condition

$$
\phi^{2}\left(\nabla_{X} Q\right) Y=0
$$

for all vector fields $X, Y \in \chi(M)$. If $X$ and $Y$ are orthogonal to $\xi$, then $M^{(2 n+1)}$ $(\phi, \xi, \eta, g)$ is said to be locally $\phi$-Ricci symmetric. It may be noted that $\phi$-symmetric implies $\phi$-Ricci symmetric, but the converse is not valid in general.

Ricci tensor $S$ of a Riemannian manifold $(M, g)$ is called $\eta$-parallel if

$$
g\left(\left(\nabla_{X} S\right) Y, Z\right)=0
$$


for all vector fields $X, Y, Z$ tangent to $M$ and orthogonal to $\xi$ where $g$ and $\nabla$ denote Riemannian metric and Riemannian connection respectively.

Ricci tensor $S$ of a Riemannian manifold $(M, g)$ is called cyclic-parallel if

$$
\left(\nabla_{X} S\right)(Y, Z)+\left(\nabla_{Y} S\right)(Z, X)+\left(\nabla_{Z} S\right)(X, Y)=0
$$

for all vector fields $X, Y, Z$ tangent to $M$. Here $\nabla$ denotes Riemannian connection.

\section{Three-dimensional $f$-Kenmotsu manifolds with Ricci soliton}

In this section we prove the following:

Theorem 3.1. In a three-dimensional $f$ Kenmotsu Ricci soliton, if $f$ is constant and the soliton vector field is Killing, then the soliton is expanding.

Proof. For a three-dimensional $f$-Kenmotsu manifold, from (2.7), we get

$$
Q X=\left(\frac{r}{2}+f^{2}+f^{\prime}\right) X-\left(\frac{r}{2}+3 f^{2}+3 f^{\prime}\right) \eta(X) \xi .
$$

Differentiating covariantly along $Y$ and using (2.4) and (2.12) we obtain

$$
\begin{aligned}
\left(\nabla_{Y} Q\right) X= & \left(\frac{d r(Y)}{2}+2 f d f(Y)+d f^{\prime}(Y)\right) X+\left(\frac{r}{2}+f^{2}+f^{\prime}\right) \nabla_{Y} X \\
- & \left(\frac{d r(Y)}{2}+6 f d f(Y)+3 d f^{\prime}(Y)\right) \eta(X) \xi \\
- & \left(\frac{r}{2}+3 f^{2}+3 f^{\prime}\right) f g(\phi X, \phi Y) \xi-\left(\frac{r}{2}+3 f^{2}+3 f^{\prime}\right) \\
& \eta(X) f(Y-\eta(Y) \xi) .
\end{aligned}
$$

Taking inner product of ( 3.2$)$ with $Y$ we have

$$
\begin{aligned}
g\left(\left(\nabla_{Y} Q\right) X, Y\right) & =\left(\frac{d r(Y)}{2}+2 f d f(Y)+d f^{\prime}(Y)\right) g(X, Y) \\
& +\left(\frac{r}{2}+f^{2}+f^{\prime}\right) g\left(\nabla_{Y} X, Y\right) \\
& -\left(\frac{d r(Y)}{2}+6 f d f(Y)+3 d f^{\prime}(Y)\right) \eta(X) \eta(Y) \\
& -\left(\frac{r}{2}+3 f^{2}+3 f^{\prime}\right) f g(\phi X, \phi Y) \eta(Y) \\
& -\left(\frac{r}{2}+3 f^{2}+3 f^{\prime}\right) \eta(X) g(Y, Y) f \\
& +\left(\frac{r}{2}+3 f^{2}+3 f^{\prime}\right) \eta(X)(\eta(Y))^{2} f .
\end{aligned}
$$

Let $\left\{e_{1}, e_{2}, \xi\right\}$ be an orthonormal $\phi$-basis at any point of a tangent space. It is known that

$$
\operatorname{div}(Q) X=g\left(\left(\nabla_{e_{1}} Q\right) X, e_{1}\right)+g\left(\left(\nabla_{e_{2}} Q\right) X, e_{2}\right)+g\left(\left(\nabla_{e_{3}} Q\right) X, e_{3}\right) .
$$


Using (3.3) in (3.4) we get

$$
\begin{aligned}
\operatorname{div}(Q) X & =\left(\frac{d r\left(e_{1}\right)}{2}+2 f d f\left(e_{1}\right)+d f^{\prime}\left(e_{1}\right)\right) g\left(X, e_{1}\right) \\
& +\left(\frac{r}{2}+f^{2}+f^{\prime}\right) g\left(\nabla_{e_{1}} X, e_{1}\right) \\
& -\left(\frac{d r\left(e_{2}\right)}{2}+6 f d f\left(e_{2}\right)+3 d f^{\prime}\left(e_{2}\right)\right) g\left(X, e_{2}\right) \\
& +\left(\frac{r}{2}+3 f^{2}+3 f^{\prime}\right) g\left(\nabla_{e_{2}} X, e_{2}\right) \\
& +\left(\frac{d r(\xi)}{2}+2 f d f(\xi)+d f^{\prime}\right) \eta(X) \\
& +\left(\frac{r}{2}+f^{2}+f^{\prime}\right) g\left(\nabla_{\xi} X, \xi\right) \\
& -\left(\frac{d r(\xi)}{2}+2 f d f(\xi)+d f^{\prime}\right) \eta(X)
\end{aligned}
$$

We know that $\operatorname{div}(Q) X=\frac{1}{2} d r(X)$. Putting $X=\xi$ in (3.5) we obtain

$$
\frac{1}{2} d r \xi=2\left(\frac{r}{2}+f^{2}+f^{\prime}\right) f-4 f d f(\xi)-2 d f^{\prime}(\xi) .
$$

If $f$-Kenmotsu manifold admits Ricci soliton then

$$
S(X, Y)=-\frac{1}{2}\left(\left(\mathcal{L}_{V} g\right)(X, Y)-\lambda g(X, Y)\right) .
$$

If $V$ is a Killing vector field, from (3.7) we get $r=-3 \lambda=$ constant. Therefore, from (3.6)

$$
\left(\frac{r}{2}+f^{2}+f^{\prime}\right) f=2 f d f(\xi)-d f^{\prime}(\xi)
$$

If $f$ is a non-zero constant then

$$
r=-2 f^{2} .
$$

Consequently, $\lambda=\frac{2}{3} f^{2}$. This completes the proof.

We know from [6] that a three-dimensional non cosymplectic $f$-Kenmotsu manifold $M^{3}(\phi, \xi, \eta, g)$ with $f$ being constant, is locally $\phi$-Ricci symmetric if and only if the scalar curvature is constant. So we get the following corollary

Corollary 3.1. If a three-dimensional $f$-Kenmotsu manifold with constant $f$ admits a Ricci soliton with Killing soliton vector field, then it is $\phi$-Ricci symmetric, and hence $\phi$-symmetric.

Again we know from [6] that in a three-dimensional non cosymplectic $f$-Kenmotsu manifold $M^{3}(\phi, \xi, \eta, g)$ with $f$ being constant, the Ricci tensor is $\eta$-parallel if and only if the scalar curvature is constant. Hence we get 
Corollary 3.2. If a three-dimensional $f$-Kenmotsu manifold with constant $f$ admits Ricci soliton with Killing soliton vector field, then its Ricci tensor is $\eta$-parallel.

From [6] we know that a three-dimensional non cosymplectic $f$-Kenmotsu manifold $M^{3}(\phi, \xi, \eta, g)$ with $f$ being constant, satisfies cyclic parallel Ricci tensor if and only if the scalar curvature is constant. So, we can state the following:

Corollary 3.3. If a three-dimensional $f$-Kenmotsu manifold with constant $f$ admits Ricci soliton with Killing soliton vector field, then its Ricci tensor is cyclic parallel.

\section{Example}

Example 4.1. Let $M=\left\{(u, v, w) \in R^{3}: u, v, w(\neq 0) \in R\right\}$ be a Riemannian manifold, where $(u, v, w)$ denotes the standard coordinates of a point in $R^{3}$. Let us suppose that

$$
e_{1}=3 w \frac{\partial}{\partial u}, \quad e_{2}=3 w \frac{\partial}{\partial v}, \quad e_{3}=-3 w \frac{\partial}{\partial w}
$$

are three linearly independent vector fields at each point of $M$ and therefore it forms a basis for the tangent space $\chi(M)$. We also define the Riemannian metric $g$ of the manifold $M$ given by

$$
g=\frac{1}{w^{2}}[d u \odot d u+d v \odot d v+d w \odot d w] .
$$

Let $\eta$ be the one form satisfying

$$
\eta(U)=g\left(U, e_{3}\right)
$$

for any $U \in \chi(M)$ and let $\phi$ be the $(1,1)$ tensor field defined by $\phi e_{1}=-e_{2}$, $\phi e_{2}=e_{1}, \phi e_{3}=0$. By the linear properties of $\phi$ and $g$, we can easily verify the following relations

$$
\begin{gathered}
\eta\left(e_{3}\right)=1, \quad \phi^{2}(U)=-U+\eta(U) e_{3} \\
g(\phi U, \phi V)=g(U, V)-\eta(U) \eta(V)
\end{gathered}
$$

for arbitrary vector fields $U, V \in \chi(M)$. This shows that $\xi=e_{3}$ the structure $(\phi, \xi, \eta, g)$ defines an almost contact metric structure on $M$. If $\nabla$ is the Livi-Civita connection with respect to the Riemannian metric $g$, then with the help of above, we can easily calculate that

$$
\left[e_{1}, e_{2}\right]=0, \quad\left[e_{1}, e_{3}\right]=3 e_{1}, \quad\left[e_{2}, e_{3}\right]=3 e_{2} .
$$

Now we recall Koszul's formula as

$$
\begin{aligned}
2 g\left(\nabla_{U} V, W\right) & =U(g(V, W))+V(g(W, X))-W(g(U, V)) \\
& -g(U,[V, W])-g(V,[U, W])+g(W,[U, V])
\end{aligned}
$$


for arbitrary vector fields $U, V, W \in \chi(M)$. Making use of Koszul's formula, we get the following:

$$
\begin{array}{ccc}
\nabla_{e_{2}} e_{3}=3 e_{2} & \nabla_{e_{2}} e_{2}=3 e_{3} & \nabla_{e_{2}} e_{1}=0 \\
\nabla_{e_{3}} e_{3}=0 & \nabla_{e_{3}} e_{2}=0 & \nabla_{e_{3}} e_{1}=0 \\
\nabla_{e_{1}} e_{3}=3 e_{1} & \nabla_{e_{1}} e_{2}=0 & \nabla_{e_{1}} e_{1}=3 e_{3} .
\end{array}
$$

From the above calculation, it is clear that $M$ satisies the condition $\nabla_{U} \xi=$ $f\{U-\eta(U) \xi\}$ for $e_{3}=\xi$, where $f=3$ is a non-zero constant. Thus we conclude that $M$ leads to an $f$-Kenmotsu manifold. Also $f^{2}+f^{\prime}$ is non-zero. This implies that $M$ is a three-dimensional regular $f$-Kenmotsu manifold. We find the components of curvature tensor and Ricci tensor as follows:

$$
\begin{gathered}
R\left(e_{2}, e_{3}\right) e_{3}=-3 e_{2}, \quad R\left(e_{3}, e_{2}\right) e_{2}=-3 e_{3}, \\
R\left(e_{1}, e_{3}\right) e_{3}=-3 e_{1}, \quad R\left(e_{3}, e_{1}\right) e_{1}=-3 e_{3}, \\
R\left(e_{1}, e_{2}\right) e_{2}=-3 e_{1}, \quad R\left(e_{1}, e_{2}\right) e_{3}=0, \\
R\left(e_{2}, e_{1}\right) e_{1}=-3 e_{2}, \quad R\left(e_{3}, e_{1}\right) e_{2}=0, \\
S\left(e_{1}, e_{1}\right)=-6, \quad S\left(e_{2}, e_{2}\right)=-6, \quad S\left(e_{3}, e_{3}\right)=-6, \\
S\left(\phi e_{1}, \phi e_{1}\right)=-6, \quad S\left(\phi e_{2}, \phi e_{2}\right)=-6, \quad S\left(\phi e_{3}, \phi e_{3}\right)=-0,
\end{gathered}
$$

$S\left(\phi e_{i}, \phi e_{j}\right)=0$ for all $i, j=1,2,3(i \neq j)$. From the above consequence, it is clear that $\phi^{2}\left\{\left(\nabla_{U} Q\right)(V)\right\}=0$ for all vector fields $U, V \in \chi(M)$. Hence $M$ is locally $\phi$-Ricci symmetric. From above we get $r=-18$, this implies the scalar curvature is constant. Moreover, $\left(\nabla_{X} S\right)\left(\phi e_{i}, \phi e_{j}\right)=0$ for $X \in \chi(M) i, j=1,2,3$. So $M$ is $\eta$-parallel, cyclic parallel. This example is also satisfying the Ricci soliton equation if $\lambda=6$. Hence $\lambda=\frac{2}{3} f^{2}$ is verified. So the soliton is expanding. Thus, Theorem 3.1 and the associated corollaries are verified by this example. 


\section{REF E R E N C E S}

1. D. E. Blair, Contact Manifolds in Riemannian Geometry, Lecture Notes in Math. $\mathbf{5 0 9}$ (1976), Springer-Verlag.

2. U. C. De and A. Sarkar, On $\phi$-Ricci symmetric Sasakian manifolds, Proceding of the Jangjeon Mathematical society, 11 (2008), 47-52.

3. U. C. De and A. Sarkar, On three-dimensional quasi-Sasakian manifolds, SUT Journal of Mathematics, 45 (2009), 59-71.

4. A. Ghosh, Certain contact metric as Ricci almost solitons, Results Math, 65 (2014), 81-94.

5. R. S. Hamilton, Ricci flow on surfaces, Contemp. Math, 71 (1988), 237-261.

6. S. K. Hui, Almost conformal Ricci solitons on $f$-Kenmotsu manifolds, Khayyam Journal of Mathematics, 5 (2019), 89-104.

7. D. Janssens and L. Vanhecke, Almost cotact structures and curvature tensor, Kodai Math. J, 4 (1981), 1-27.

8. K. Kenmotsu, A class of almost contact Riemannian manifolds, Tohoku Math. Journal, 24 (1972), 93-103.

9. Z. Olszak, Locally conformal almost cosympletic manifolds, Colloq. Math. 57 (1989), 73-87.

10. Z. Olszak, Rosca, R., Normal locally conformal almost cosympletic manifolds, Publ. Math. Debrecen 39 (1991) 315-323.

11. G. Perelman, The entropy formula for the Ricci flow and its geometric applications, arXiv: 0211159 mathDG, (2002)(Preprint).

12. S. Pigola et al., Ricci almost solitons, Ann. Sc. Norm. Sup. Pisa Cl. Sci, 10(2011), 757-799.

13. A. Sarkar, A. Sil and A. K. Paul, Ricci almost soliton on three-dimensional quasiSasakian manifold, Proc. Natl. Acad. Sci., India, Sect. A Phys. Sci, 89(2019), 705-710.

14. A. Sarkar and P. Bhakta, Ricci almost soliton on $(\kappa, \mu)$ space forms, Acta Universitatis Apulensis, 57(2019), 75-85.

15. A. Sarkar, A. Sil and A. K. Paul, Ricci soliton on three dimensional trans Sasakian manifold and Kagan Subprojective spaces, Eukrainian Math Journal, 72(2020), 488494.

16. R. Sharma, Almost Ricci solitons and K-contact geometry. Montash Math., 175 (2014), 621-628.

17. T. Takahashi, Sasakian $\phi$-symmetric spaces, Tohoku Math. J, 29 (1977), 91-113.

Avijit Sarkar

Department of Mathematics

University of Kalyani

Kalyani 741235

West Bengal

India

avjaj@yahoo.co.in 
Pradip Bhakta

Department of Mathematics

University of Kalyani

Kalyani 741235

West Bengal

India

pradip020791@gmail.com 